\title{
Reference values for ductus venosus flow in normal gestation among an Egyptian population
}

\author{
Ashraf Anas Zytoon", Nada Nabil Ahmed El-Abakawy² and Shaimaa Abdel-hamid Hassanein ${ }^{1 *}$ (D)
}

\begin{abstract}
Background: Doppler studies of the fetal circulation have demonstrated the importance of the ductus venosus in the continuous monitoring of fetuses, especially those of high-risk pregnancies that can lead to abnormal pregnancy outcome. We tried to put reference values for the normal ductus venosus (DV) flow in normal gestation to be used in further studies assessing the different pathologies.

Results: The DV length ranged from 9.8 to $20.9 \mathrm{~mm}$, and the diameter ranged from 0.6 to $2.3 \mathrm{~mm}$. The DV pulsatility index, resistive index, and systolic velocity/maximum velocity during atrial wave ratio declined linearly with increasing of gestational age and were increased after that during the last weeks of pregnancy. Ductus venosus S wave and A wave velocities increased linearly from a mean of 38.30 at the first trimester to 61.68 at the third trimester and from a mean of 11.38 at the first trimester to 30.71 at the third trimester respectively.

Conclusion: A reference range of the normal ductus venosus different Doppler parameters was constructed in a centile module.
\end{abstract}

Keywords: Ductus venosus, Doppler measures, Reference values, Normal gestation

\section{Background}

The ductus venosus (DV) is a tiny vein connecting the umbilical circulation directly to the inferior vena cava where $20-30 \%$ of the highly oxygenated blood from the placenta bypasses the hepatic circulation and flows through the ductus venosus directly towards the foramen ovale into the left atrium [1].

Ductus venosus has characteristic wave forms characterized by two peaks during systole ( $\mathrm{S}$ wave) and diastole (D wave). In contrast to the neighboring inferior vena cava and hepatic veins, the blood flow normally remains orthograde during atrial contraction giving another peak during atrial contraction in late diastole (A wave) $[2,3]$.

The phases of the DV flow-velocity waveform are related in timing to the phases of the cardiac cycle and concurrent volume and pressure changes in the cardiac chambers. Thus, changes of the venous flow-velocity

\footnotetext{
* Correspondence: shaimaahamid@hotmail.com; shaimaa.abd.alhameed66@med.menofia.edu.eg

${ }^{1}$ Radiology Department, Faculty of Medicine, Menoufia University, Yassin

Abdel-Ghafar Street, Shebin El-Kom, Menoufia, Egypt

Full list of author information is available at the end of the article
}

profile may be interpreted to reflect cardiac function. However, although widely considered a parameter of cardiac preload, DV Doppler indices do not show reproducible relationships with parameters of cardiac function in normal and high-risk pregnancies $[4,5]$.

One of the possible explanations is that traditional DV Doppler indices are not directly related to cardiac function. Understanding the relationship between venous flow dynamics and cardiac function is of critical importance to reach an accurate interpretation of Doppler findings [6].

Doppler studies of the fetal circulation have demonstrated the importance of the DV in the continuous monitoring of fetuses with intrauterine growth restriction due to placental insufficiency, with some reports demonstrating an association of abnormal DV flow patterns and fetal chromosomal abnormalities, congenital cardiac defects, or adverse pregnancy outcome in highrisk pregnancies where increased values of the pulsatility index for veins (PIV) and absent or reversed flow during atrial contraction were related to abnormal pregnancy outcome [7-9]. 
In this study, we tried to put reference values for the normal DV flow in normal gestation to be used in further studies assessing the different pathologies.

\section{Methods}

\section{Patients}

This was a longitudinal multi-centric study including 230 low-risk singleton pregnancies. Doppler ultrasound examination of the DV was carried out between the 11th and 40th week of gestation, with more than one assessment of Doppler examination, and was performed by one radiologist. The research protocol was approved by the local Ethics Committee following the Helsinki Declaration with informed written consent obtained from every subject involved in the study prior to the examination.

Gestational age was confirmed by first-trimester crown-rump length measurement or assessment of head biometry (BPD and $\mathrm{HC}$ ), abdominal circumference (AC), and femur length (FL) at second and third trimesters. The ultrasound examinations were predominantly done transabdominally: only in the case of suboptimal abdominal access that the examination was performed transvaginally especially in those at the late first trimester, and this was performed in 10 cases.

Patient selection was based on certain inclusion criteria including no risk pregnancy, singleton pregnancy, accurate gestational age based on the last menstruation date adapted with ultrasound parameters, gestational age between 11 and 40 weeks, normal fetal growth (between 10th and 90th percentiles of the growth chart), normal amniotic fluid volumes, and normal resistance indices in the umbilical artery and the middle cerebral artery.

Those with the following criteria were excluded: multiple pregnancies, fetal malformation or chromosomal abnormalities, maternal chronic disease (hypertension, diabetes mellitus, rheumatic or autoimmune disease), hydrops fetalis and consumption of hormonal contraceptive agents, IUGR (birth weight of less than 10th percentile), macrosomia (birth weight of more than 90th percentile), labor activity, and satisfactory DV Doppler waveform could not be obtained.

The study population of 230 women (690 observations as DV examined at least three times) was recruited from the normal pregnancy population to a prospective longitudinal observational study of the ductus venosus flow velocities and calculated.

\section{Methods}

Three measurements per session were performed for each woman. The scan was performed in a quiet room with a pleasant temperature where the patient was positioned in horizontal dorsal decubitus with a slightly right or left rotation to facilitate the evaluation, and all
Doppler measurements were obtained in the absence of maternal and fetal movements as well as of fetal breathing movements. The fetal heart rates were within a physiologic range of 120-160 bpm during the scan.

While calculating the gestational age, those at 3 days and below were taken in lower gestation week and those after 4 days and above were taken in higher gestational week; for example, a patient at 20 weeks and 2 days gestation was taken as 20 weeks and a patient at 20 weeks and 5 days gestation was taken as 21 weeks.

Doppler ultrasound measurements were recorded using the transabdominal $2-5-\mathrm{MHz}$ curved-array transducer (Philips HD9, Philips HD11, GE LOGIC P3 and GE LOGIC P5). The high-pass filter was set at $100 \mathrm{~Hz}$, and the spatial peak-temporal average intensity for color and pulsed Doppler was less than $50 \mathrm{~mW} / \mathrm{cm}^{2}$ and 100 $\mathrm{mW} / \mathrm{cm}^{2}$, respectively.

The DV Doppler waveforms were determined as follows.

The DV was visualized first by color Doppler imaging in a midsagittal view or in an oblique transverse abdominal plane to identify the vessel connecting the umbilical vein and the inferior vena cava. Color aliasing was usually found within the DV as there is an approximately two- to threefold increase in blood flow velocity at the origin of the ductus venosus from the umbilical vein.

Pulsed Doppler gate was then placed at the origin of the DV in the distal portion of the umbilical sinus where it can be visualized in its full length in a midsagittal section of the fetal trunk whether sagittal anterior or posterior positions.

If the sagittal views were not possible, then an oblique transverse section through the fetal abdomen provided a good visualization of the DV inlet. The size of the sample volumes used ranged between 2.5 and $6 \mathrm{~mm}$ per the vessel diameter to reduce the interference of velocities from the neighboring vessels. The sample volume was kept wide to ensure the recording of the maximum velocity during the cardiac cycle, usually a 1-2-mm sample gate. The color scale was then increased and then color gain reduced till the echogenic surfaces of the ductus venosus nearly displayed transparent color flow.

The ductus venosus length and diameter were recorded followed by measuring the waveforms which were traced automatically and manually, with an insonation angle $<30^{\circ}$ used to measure the DV peak systolic velocity (PSV), maximum velocity during atrial contraction (VAC), systolic velocity/maximum velocity during atrial wave ratio (S/A ratio), resistive index (RI), and pulsatility index (PI) (Fig. 1).

For reduced inter-observer variations, at least three consecutive high-quality waveforms were recorded in each fetus during quiescence and normograms were constructed from the results by using an average of three measurements of each normal fetus. 


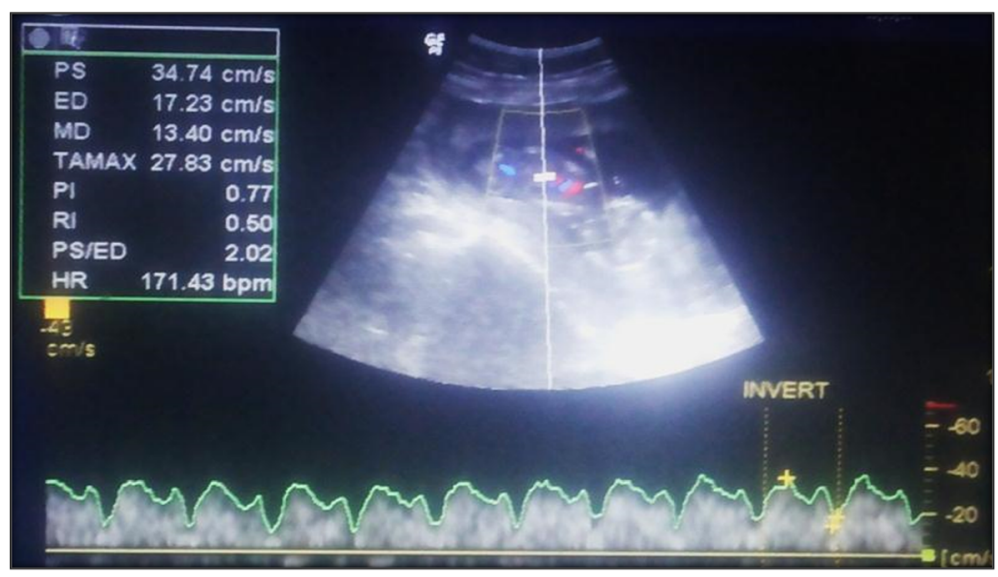

Fig. 1 A triplex image showing the DV measurement of a 22 weeks' fetus in sagittal view with the normal Doppler spectrum

\section{Statistical methods}

Statistical analysis was done using Stata $\odot$ version 15 (StataCorp LLC, College Station, TX, USA) and MedCalc@ version 18 (MedCalc@ Software bvba, Ostend, Belgium).

The methodology used to model the reference intervals for Doppler parameters on gestational age was based on the methods described by Altman (1993), Altman and Chitty (1993), and Wright and Royston (1997).

The method includes the following steps:

1. If the distribution of the measurements (the variable for which to establish a reference interval) shows skewness at different levels of gestational age, the measurements are transformed using a Box-Cox power transformation.

2. The transformed measurements are modeled on gestational age using weighted polynomial regression (Altman \& Chitty, 1994). This regression model gives the mean of the (transformed) measurements as a function of gestational age: mean (gestational age).

3. The residuals of this regression model are calculated.

4. The absolute residuals, multiplied by $\sqrt{\frac{\pi}{2}}$, are modeled on gestational age using weighted polynomial regression (Altman, 1993). This second regression model gives the standard deviation of the (transformed) measurements as a function of gestational age: SD (gestational age).

5. For every gestational age in the observed range, the reference interval is calculated by taking mean (gestational age) $\pm z \times \mathrm{SD}$ (gestational age) For a $95 \%$ reference interval, $z=1.96$. The resulting values are then back-transformed to their original scale.

\section{Results}

A total of 230 singleton pregnant women were recruited into the study with gestational ages from 11 to 40 weeks; their maternal age ranged from 18 to 45 years old with the mean age being $26.1 \pm 6.7$ years old. They were distributed as 18 pregnant females at the first trimester, 87 at the second trimester, and 125 pregnant females at the third trimester (Fig. 2). The minimum number of studied cases was at the weeks 20, 23, 26, 27, and 29 representing $4.5 \%$, while the maximum number was at week 38 representing $11.8 \%$.

The calculated reference ranges for DV length in normal pregnancies based on 690 observations with 1th-99th percentiles showed a parabolic course where the minimum length was $0.98 \mathrm{~cm}$ and the maximum length was $2.09 \mathrm{~cm}$ (from 0.98 to $1.21 \mathrm{~cm}$ with an average of $1.09 \mathrm{~cm}$ at the first trimester, from 0.98 to $1.60 \mathrm{~cm}$ with an average of $1.24 \mathrm{~cm}$ at the second trimester, and from 1.34 to $2.09 \mathrm{~cm}$ with an average of $1.67 \mathrm{~cm}$ at the third trimester).

The calculated reference ranges for DV diameter in the 690 observations also showed a parabolic curve with 1st-99th percentiles. It ranged from 0.06 to $0.16 \mathrm{~cm}$ with an average of 0.11 at the first trimester, from 0.08 to $0.18 \mathrm{~cm}$ with an average of $0.15 \mathrm{~cm}$ at the second trimester, and from 0.13 to $0.23 \mathrm{~cm}$ with an average of $0.16 \mathrm{~cm}$ at the third trimester (Fig. 3).

Regarding the Doppler measurements, we found a significant decrease in the pulsatility index (PI) parameter with increasing gestational age where it ranged from 0.84 to 1.39 with an average of 1.10 at the first trimester, from 0.74 to 1.16 with an average of 0.88 at the second trimester, and from 0.45 to 1.34 with an average of 0.75 at the third trimester; however, a separate increase in the measuring was noted at week 33 till 39.5 weeks which correlates with the 95th, 98th, and 99th percentiles (Fig. 4). 


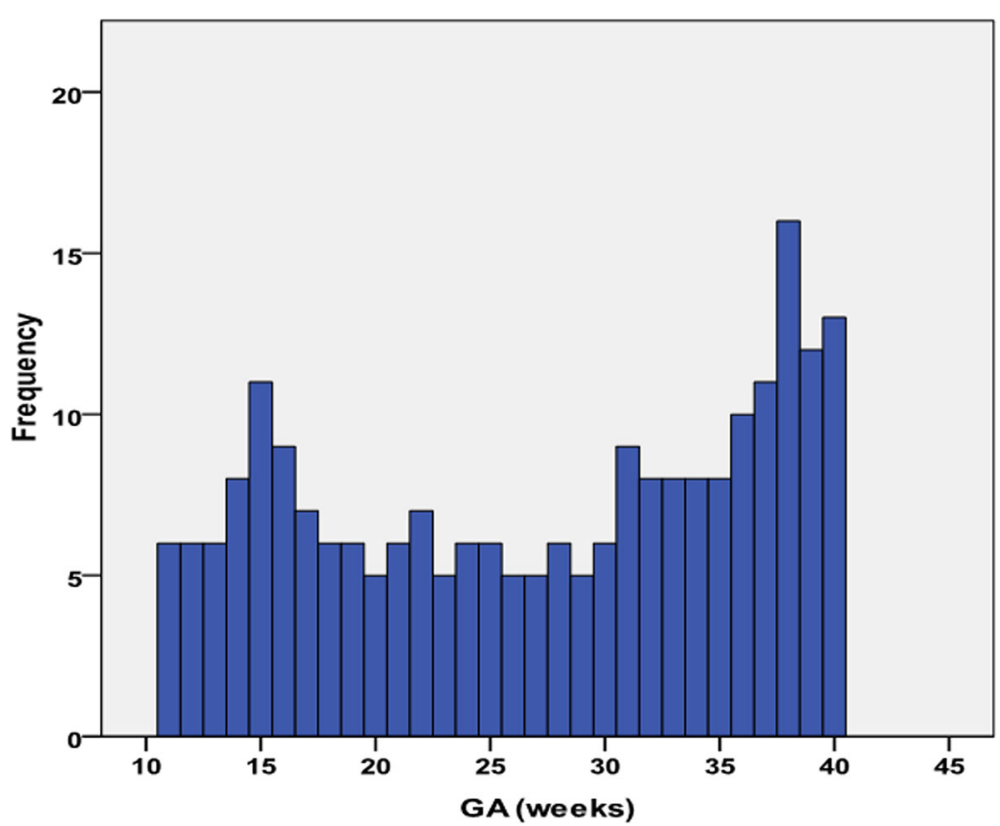

Fig. 2 A histogram showing the frequency distribution of gestational age

Resistive index (RI) also showed a similar pattern to the PI with a range of 0.62 to 0.85 with an average of 0.72 at the first trimester, from 0.55 to 0.77 with an average of 0.54 at the second trimester, and from 0.36 to 0.83 with an average of 0.53 at the third trimester. A similar increase was also noted at weeks 34.5 till 39.5 corresponding at 95\%, 98\%, and 99\% percentile (Fig. 5).
The $\mathrm{S}$ wave velocity on the other hand showed a significant increase with increasing gestational age. It ranged from 11.17 to $69.66 \mathrm{~cm} / \mathrm{s}$ with an average of $38.30 \mathrm{~cm} / \mathrm{s}$ at the first trimester, from 13.78 to $97.11 \mathrm{~cm} /$ $\mathrm{s}$ with an average of 51.40 at the second trimester, and from 27.77 to $94.27 \mathrm{~cm} / \mathrm{s}$ with an average of 61.68 at the third trimester. However, there was another reduction of

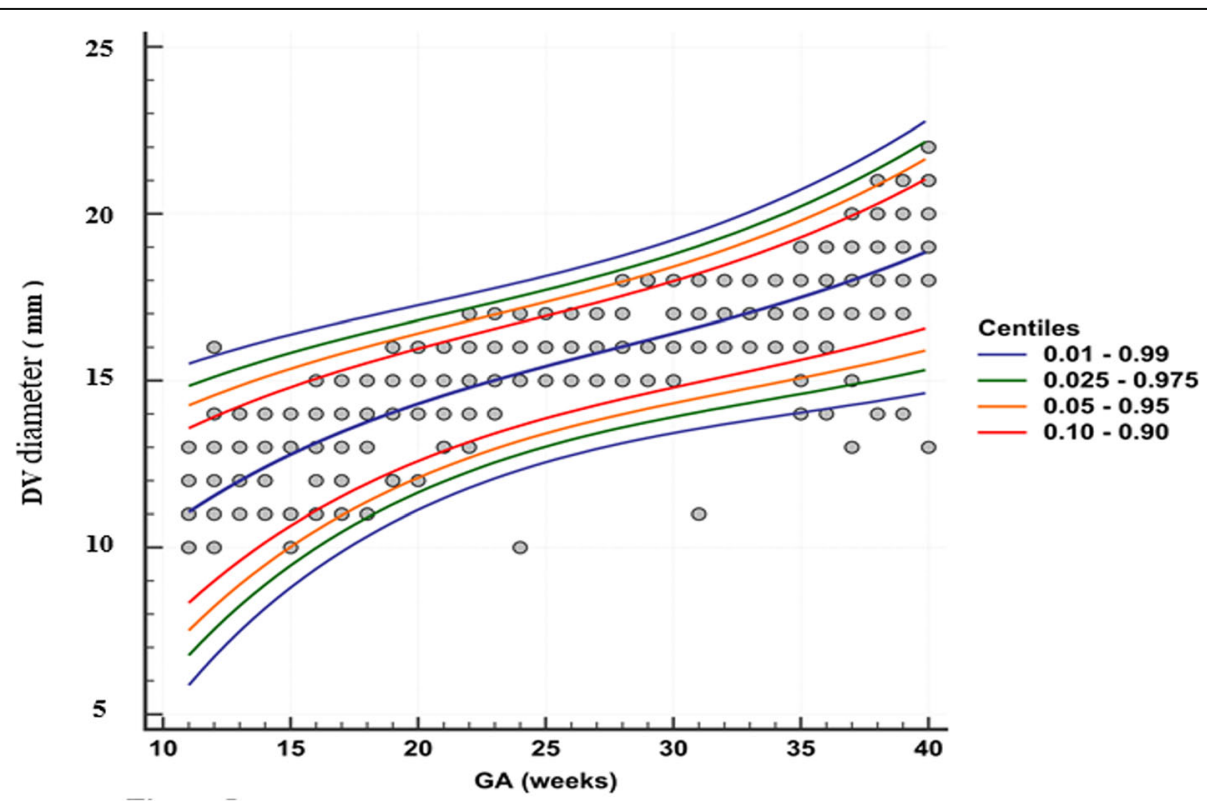

Fig. 3 Scattered diagram of gestational age individual measurement and calculated reference ranges for DV diameter in normal pregnancies based on 690 observations with 1th-99th percentiles (blue lines) 


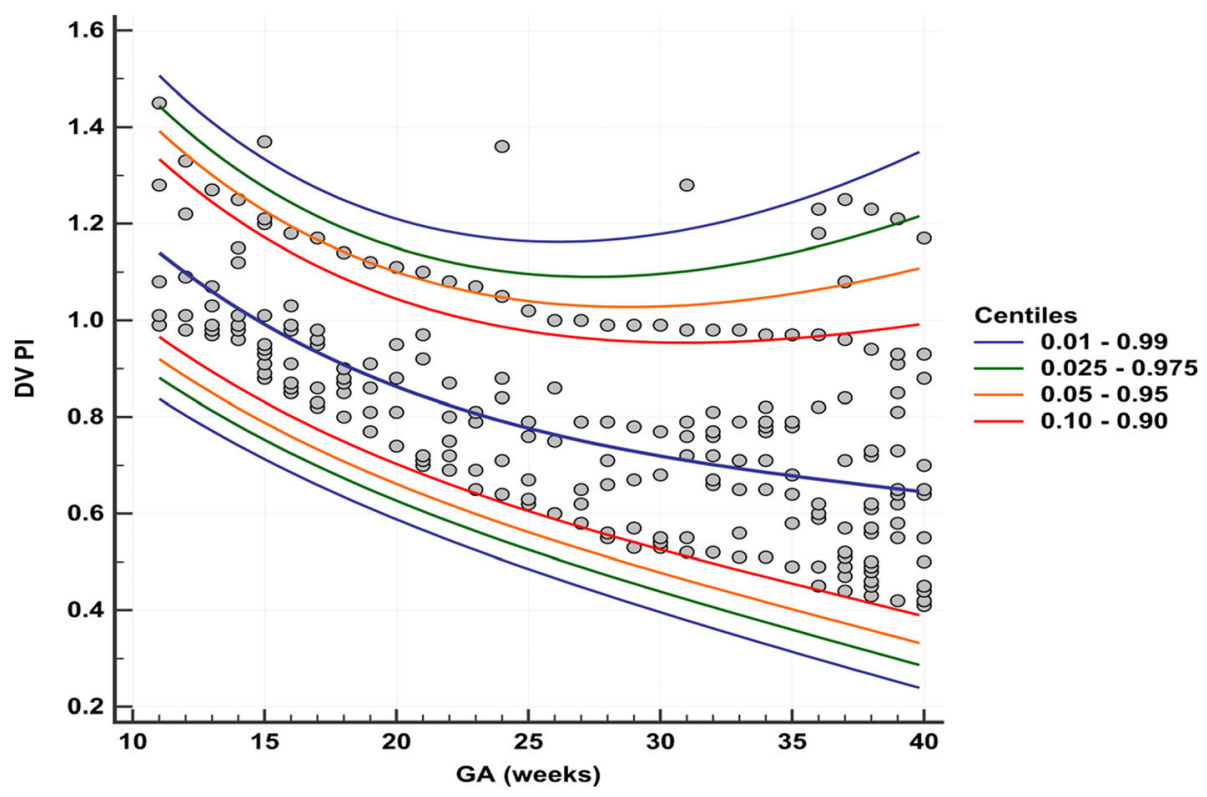

Fig. 4 Scattered diagram of gestational age individual measurement and calculated reference ranges for DV PI in normal pregnancies (690 observations) with 1th-99th percentiles (blue lines)

the measuring parameter at 32.5 till 39.5 weeks corresponding to 95th, 98th, and 99th percentiles (Fig. 6).

The calculated reference ranges for DV A wave velocity in normal pregnancies (690 observations) from 1th to 99th percentiles showed a significant increase in the A wave velocity with increasing gestational age. It ranged from 1.61 to $25.29 \mathrm{~cm} / \mathrm{s}$ with an average of 11.38 at the first trimester, from 2.96 to $49.42 \mathrm{~cm} / \mathrm{s}$ with an average of 21.10 at the second trimester, and from 9.65 to $56.57 \mathrm{~cm} / \mathrm{s}$ with an average of 30.71 at the third trimester (Fig. 7).

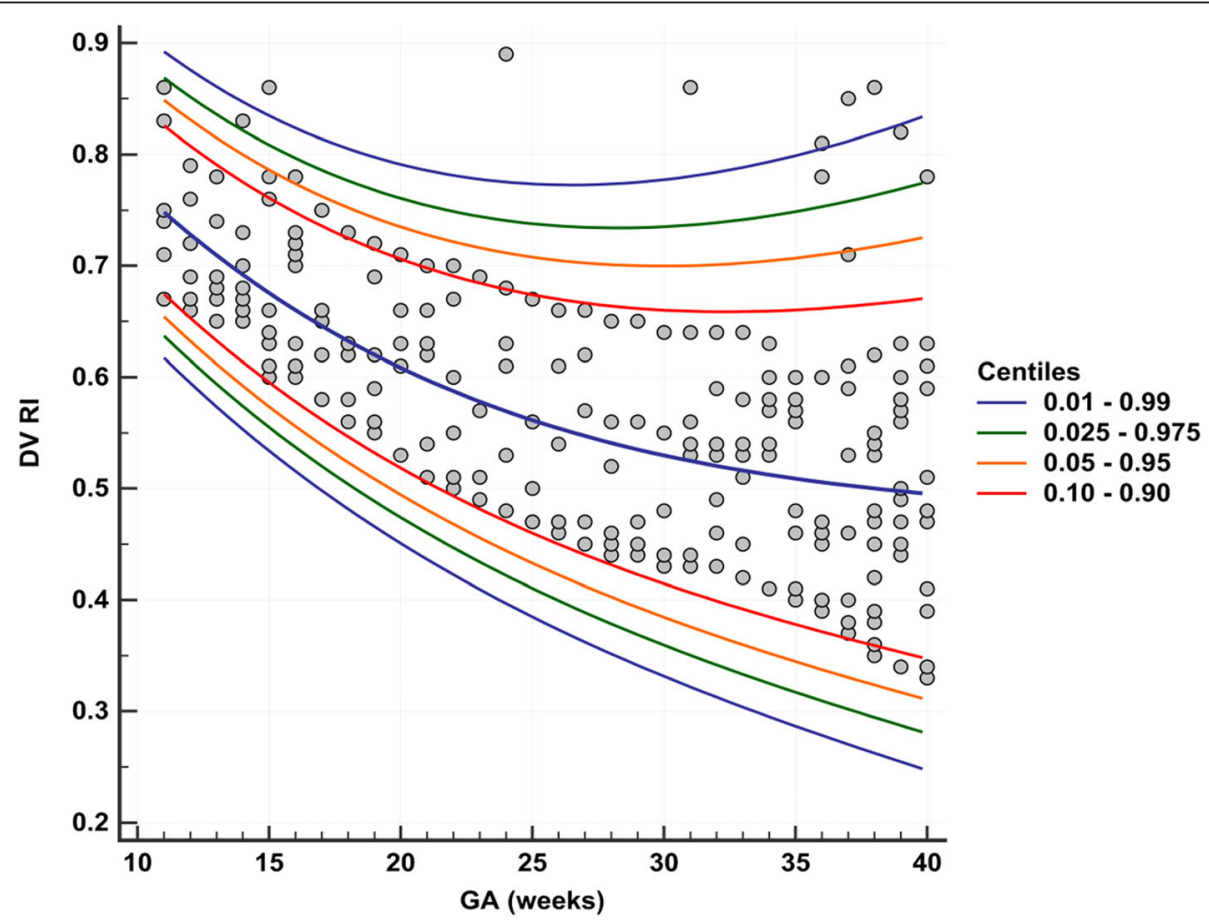

Fig. 5 Scattered diagram of gestational age individual measurement and calculated reference ranges for DV RI in normal pregnancies (690 observations) with 1th-99th percentiles (blue lines) 


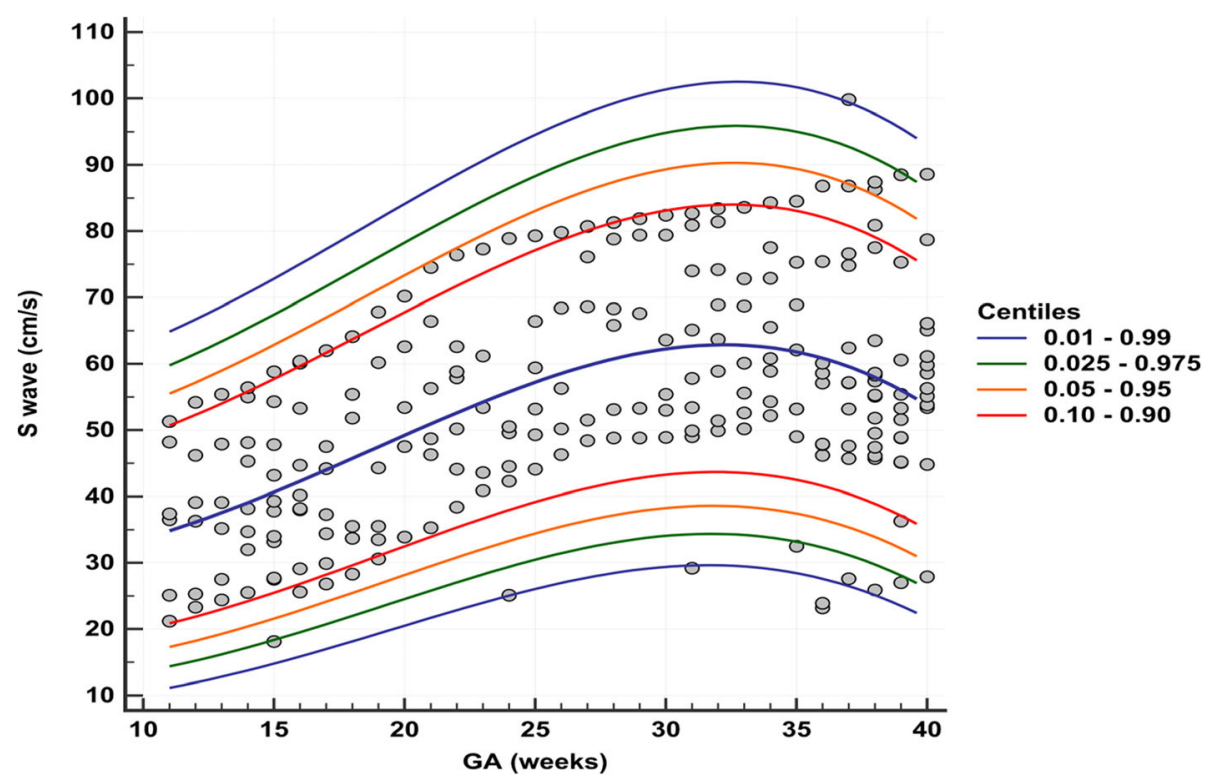

Fig. 6 Scattered diagram of gestational age individual measurement and calculated reference ranges for DV S wave velocity in normal pregnancies (690 observations) with 1th-99th percentiles (blue lines)

Consequently, the calculated reference ranges for DV $\mathrm{S} / \mathrm{A}$ ratio showed a significant decrease in the S/A ratio with increasing gestational age where it ranged from 2.47 to 5.94 with an average of 2.83 at the first trimester, from 2.11 to 4.53 with an average of 2.94 at second trimester, and from 1.54 to 5.02 with an average of 2.55 at third trimester. The measuring parameter increased again from 28 to 38 weeks at 95th, 98th, and 99th percentiles.

\section{Discussion}

The DV has a sphincter-like action. It appears as a narrow vessel projecting a high-velocity jet posteriorly to reach the foramen ovale. The high peak velocity in the DV, comparable with arterial velocities, probably gives the blood sufficient momentum to reach the foramen ovale without extensive mixing with the deoxygenated blood $[10,11]$. Velocimetry of the DV carries new diagnostic possibilities to evaluate preload or cardiac

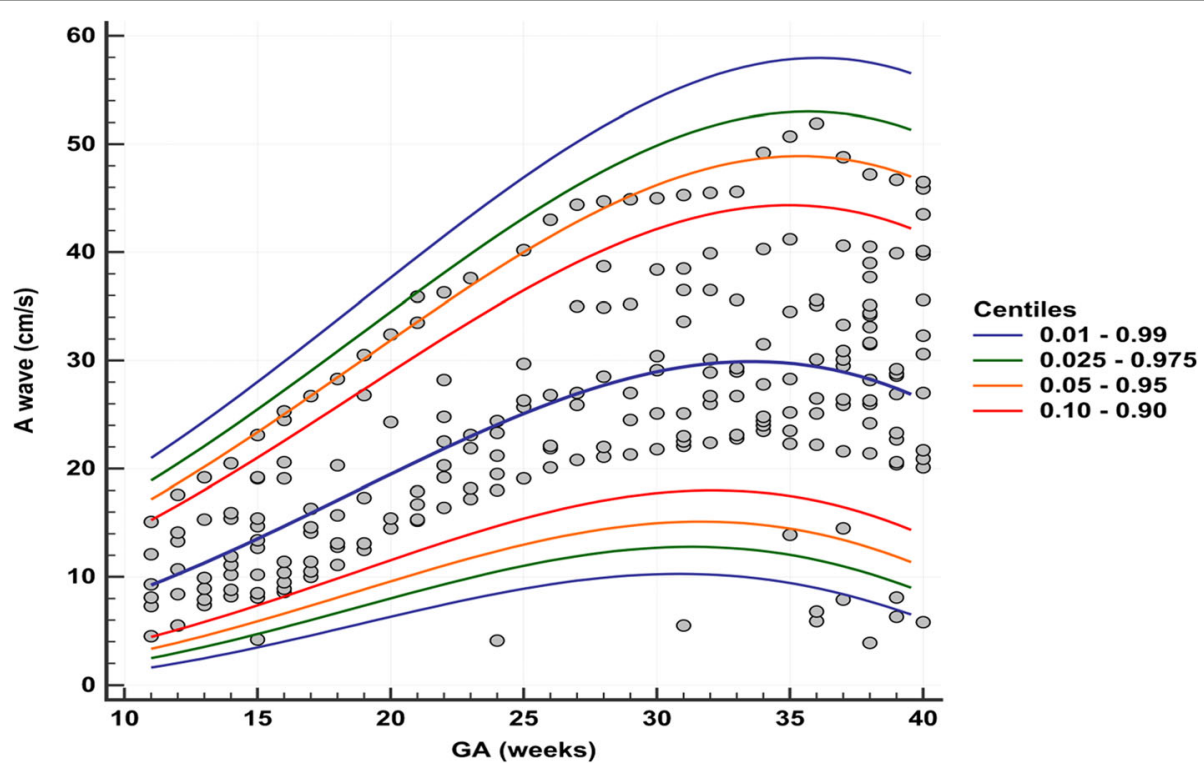

Fig. 7 Scattered diagram of gestational age individual measurement and calculated reference ranges for DV A wave velocity in normal pregnancies (690observations) with 1th-99th percentiles (blue lines) 
function. The normal Doppler flow waveform of the ductus venosus indicates the continuous triphasic forward flow throughout the cardiac cycle with a peak during systole, another one during passive diastolic filling, and a smaller nadir during atrial contraction [12, 13].

Several studies investigated the diagnostic value of ductus venosus blood flow in detection of fetuses with congenital heart disease, hypoxic or congestive fetal myocardial diseases both from a clinical and a scientific point of view. There is now a well-documented association between abnormal DV flow and complicated monochrionic multiple gestation, chromosomal anomalies, and adverse fetal outcome [1-3, 14].

Hence came the need for sitting reference values for the ductus venosus different waves. The purpose of this study was therefore to establish longitudinal reference ranges for DV PSV, VAC, S/A ratio, RI, PI, and diameter suitable for use with serial measurements for fetal surveillance, and we have also provided the necessary terms for calculating individually conditional reference intervals suitable for individual serial measurements.

The reference ranges we have established for the DV differ slightly from those of other cross-sectional studies. In comparison, the reference ranges of DV PSV curve published by Bahlmann et al. [15] were at 14 weeks 48 , at 30 weeks 65.71 , and at 40 weeks 65.83 , while our results were at 14 weeks 41.4 , at 36 weeks 62.63 , and at 40 weeks 57.11. Comparing with Axt-Fliedner et al. [16] (at 11 weeks 34.1 , at 16 weeks 45.5 , and at 20 weeks 57.5 ), our results were at 11 weeks 37.14 , at 16 weeks 44.69 , and at 20 weeks 51.58. Our findings were close to Bahlmann et al.'s [15] findings which had few observations for the last weeks of pregnancy, also close to AxtFliedner et al.'s [16] findings which had few observations in the early weeks and excluded fetuses with biometric parameters of the head and abdomen outside the $90 \%$ reference interval.
In comparison with another longitudinal study of Kessler et al. [17], the reference ranges of DV PSV were at 21 weeks 60.81, at 31 weeks 71, and remained at this level until 40 weeks) while our results were at 21 weeks 53.29, at 31 weeks 64.72 , and at 40 weeks 57.11. The authors noted a linear increase in mean peak flow in the previous studies; furthermore, our reference ranges are lower than those reported in this study, as demonstrated in Fig. 8. The observed difference may most likely be due to the different statistical methods used in establishing the reference range and to the different size of the patient population.

In comparison to our reference DV A wave velocity curve with those published by Bahlmann et al. [15], AxtFliedner et al. [16], and Kessler et al, [17], we have demonstrated a pattern for A wave velocity with some difference in ranges. Our findings were higher than Kessler et al.'s [17] findings as shown in Fig. 9; the explanation for this difference might be that Doppler traces with mono- and biphasic flow patterns with comparably high end-diastolic velocities were included in their analysis as they were considered a normal variant, while in our study these traces were excluded. In addition, differing methods for statistical analysis may account for this difference.

In our study, DV S/A ratio nomogram had a parabolic pattern close to that found by Bahlmann et al. [15] (at 20 weeks 2.58 , at 30 weeks 2.01 , and at 40 weeks 1.99 ), that is probably attributed to the fact that the Doppler standard curves of the ductus venosus described in both studies were derived from a large patient population and show greater homogeneity of the measuring values for the individual weeks of gestation.

There was a slight difference in range compared to the work of Tongprasert et al. [18] and a wide difference when compared to Axt-Fliedner et al. [16] as shown in Fig. 10. This difference is probably depending on

S Wave Velocity
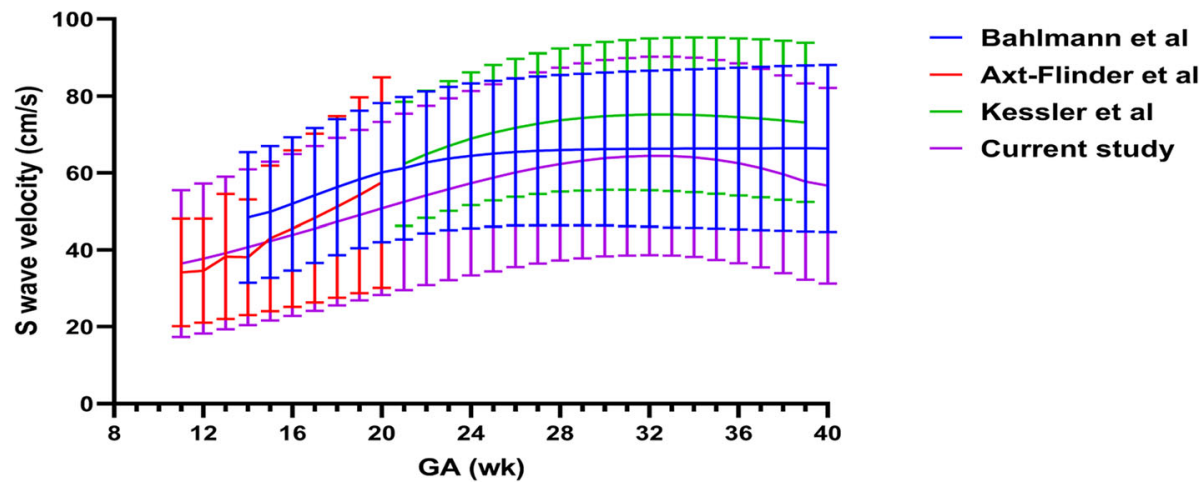

- Current study

previous studies 


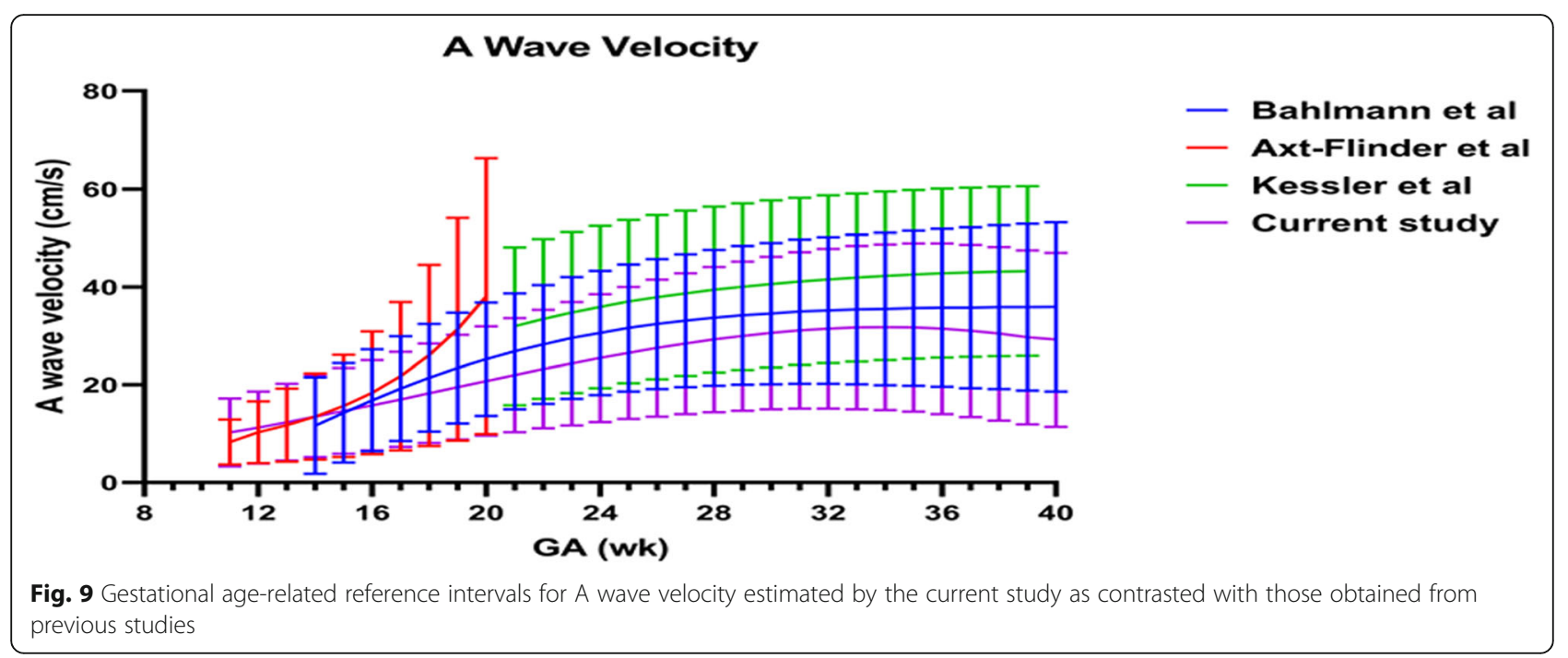

equipment, insonation techniques, angle correction, and racial factors. These findings suggest that each population group may probably need its own normal reference range for clinical application.

Regarding the DV PI curve, our references were close to those published by Bahlmann et al. [15] and Tongaprasert et al. [18]. We have demonstrated a pattern for PI with some difference in ranges; however, it was determined to be close to it. Per Bahlmann et al. [15], the calculated indices were associated with markedly greater reliability and less intra-individual variation than which of maximum flow velocities, and we have demonstrated that their PI pattern was like our present results at midgestation, but lower than our results later in pregnancy, as shown in Fig. 11. The difference may be due to that reference curves were constructed for individual measuring parameters based on growth function from a fourparameter class of monotonic continuous functions according to the smallest square principle. And the same
Doppler velocimetry technique used by this study was an explanation of the similarity.

Tongaprasert et al. [18] on the other hand found that the DV indices decreased relatively rapidly at the first half of pregnancy and were relatively constant or slightly decreased during the second half of pregnancy. Therefore, the linear equation could not be used in evaluating DV velocity in the first half of pregnancy, and this may be the cause that our present results were higher than those obtained by that study. The measurement of DV indices can be achieved in most women both in early and late pregnancy, and reproducibility is well acceptable as indicated by inter- and intra-observer variations may be another explanation.

Our findings were in close relation with Axt-Fliedner et al.'s [16] findings that were characterized by a parabolic pattern; the similarity with their result was probably due to the use of the same insonation angle correction.

\section{S/A Ratio}

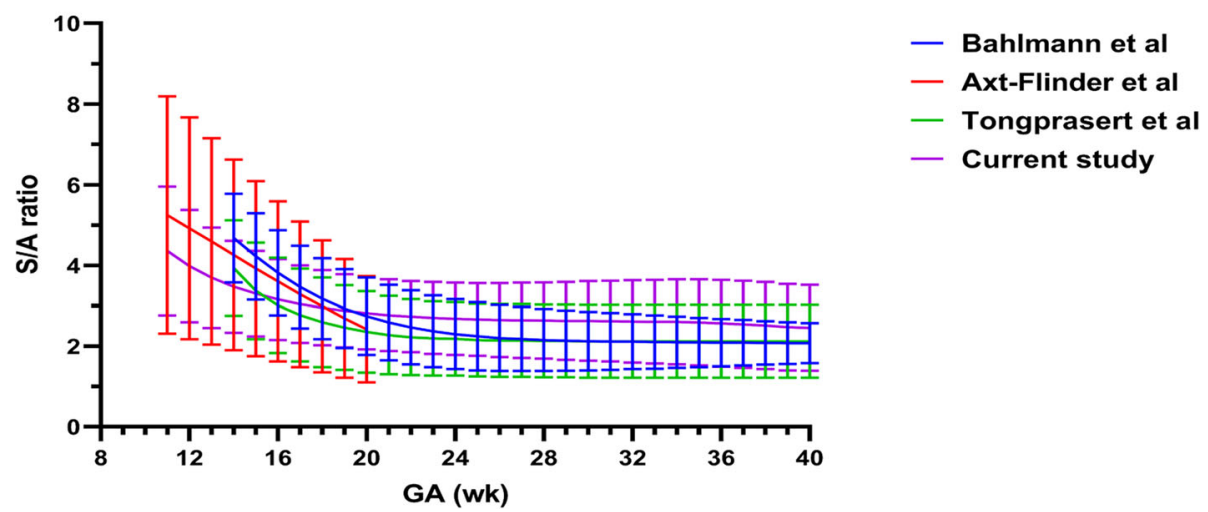

Fig. 10 Gestational age-related reference intervals for S/A ratio estimated by the current study as contrasted with those obtained from previous studies 
DV PI

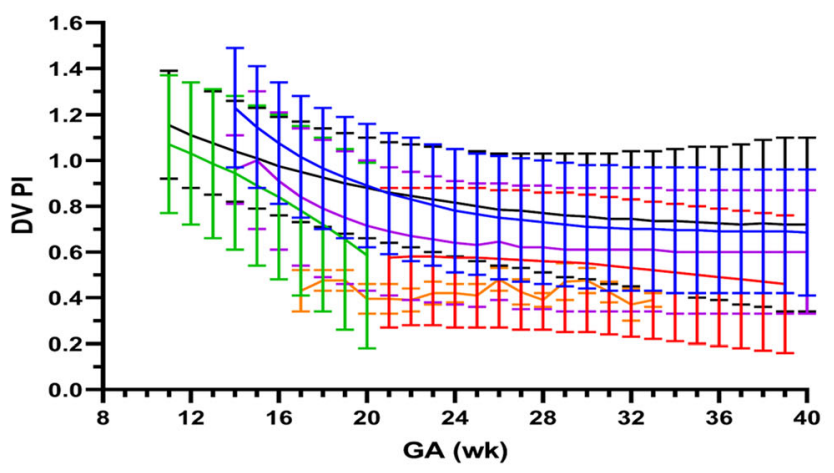

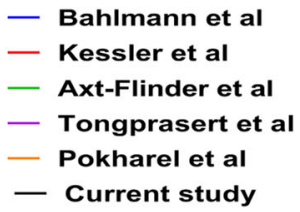

- Bahlmann et al

- Kessler et al

- Tongprasert et al

- Current study
On the other hand, our references were higher than those obtained by Kessler et al. [17] and Pokharel et al. [19]. The difference was attributed to the fact that the former study showed a smaller sample size than our study (160 pregnancies) and the latter study did not correlate the pulsatility index with the gestational age with scattered distribution; however, their values were below 1.0 in all gestational ages.

Bahlmann et al. [15] found that the reference curve for the RI was characterized by a parabolic pattern (at 14 weeks 0.67 , at 30 weeks 0.48 , and at 40 weeks 0.46 ). AxtFliedner et al. [16] also showed similar results (at 11 weeks 0.77 , at 14 weeks 0.69 , and at 20 weeks 0.51 ), while our results were at 11 weeks 0.75 , at 14 weeks 0.69 , at 20 weeks 0.61 , at 30 weeks 0.54 , and at 40 weeks 0.52 .

Our findings were in close relation with Bahlmann's findings especially during 16-24 weeks, but higher than those later in pregnancy. It was demonstrated that our reference ranges were lower than those obtained by AxtFliedner et al. (Fig. 12); in their study, the ratios were calculated from the respective ductus venosus flow velocities; different statistical analysis and different sample size may explain the different results.

Comparing our reference ranges of DV diameter curve with those published by Pokharel et al. [19], our results were close to each other (their results were 1.08 at 17 weeks, 1.44 at 25 weeks, and 1.82 at 33 weeks) while our results are at 17 weeks 1.13 , at 25 weeks 1.40 , and at 33 weeks 1.71 .

Our longitudinal reference ranges for the DV velocities and indices are suitable both for single observations and for serial measurements (when using the corresponding terms). Analysis of the numerical values of the parameters assessed showed similarities and disagreements compared with other studies conducted on normal pregnancies.

In general, the differences observed could have been due to the different size and age of the patient population, statistical analysis, and design of each study. And some variation in reference ranges is seen, probably

\section{RI}
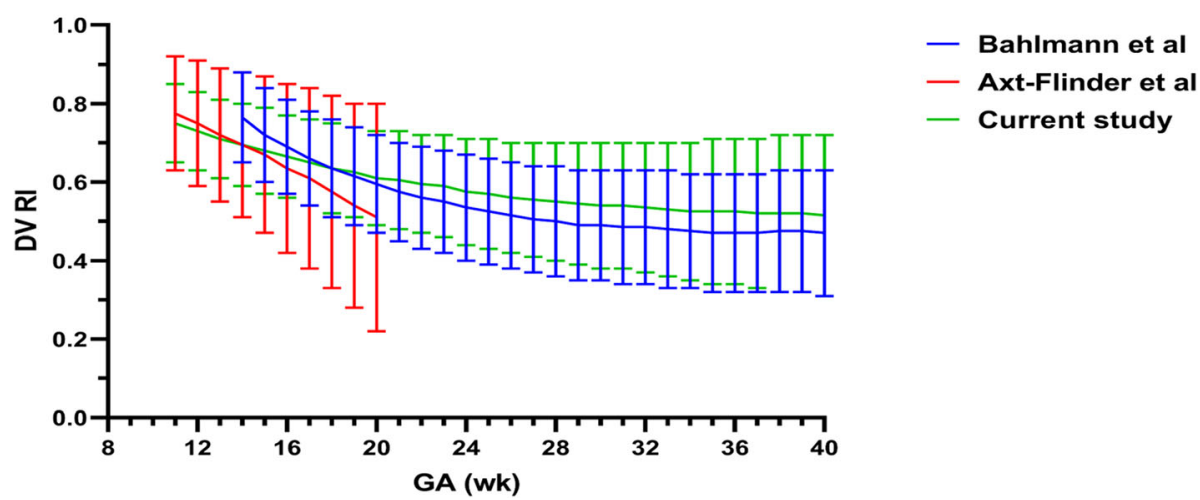

- Current study

Fig. 12 Gestational age-related reference intervals for DV RI estimated by the current study as contrasted with those obtained from previous studies 
depending on the equipment, sonologist experience, insonation techniques, angle correction, and positioning of the sample volume of pulsed Doppler in the DV.

The strengths of this study are (1) the adequate sample size for each gestational week; (2) large numbers of observations, each case was examined at least three times; and (3) that we also included fetuses in the late first trimester and early second trimester [11-19], unlike most other studies. Moreover, inter-observer variations of the measurement are acceptable.

The limitation of the present study can be multiple. The error of judgment of the radiologist during the study period cannot be excluded. The miniature size of the ductus venosus, habits of the patient while doing the procedure, and cooperativity of the patient during the study period can affect the accuracy of the results of the study.

The technical pitfalls in DV measurement should be mentioned here: this measurement is occasionally time consuming and not always simple, especially when fetuses are in an improper position.

Our longitudinal reference ranges for the DV velocities and indices are suitable both for single observations and for serial measurements (when using the corresponding terms). Analysis of the numerical values of the parameters assessed showed similarities and disagreements compared with other studies conducted on normal pregnancies.

\section{Conclusion}

Ductus venosus Doppler indices are raising great concern in the follow-up of the fetuses, especially those of high-risk pregnancies and suspected chromosomal or congenital anomalies. Hence, the need of sitting normal range is of great importance. We tried to put a reference range based on a centile module for our population.

\section{Abbreviations}

AC: Abdominal circumference; BPD: Bi-parietal diameter; DV: Ductus venosus; FL: Femur length; HC: Head circumference; PI: Pulsatility index; RI: Resistive index

\section{Acknowledgements}

The authors acknowledge the help of the nurses at the different ultrasound units.

\section{Authors' contributions}

AAZ gave the idea, put the study design, and revised and collected the patients' data and the results. NNE collected the data and analyzed them. $\mathrm{SAH}$ wrote and revised the paper. They all approved the final version of the manuscript.

\section{Funding}

This study had no funding from any resource.

\section{Availability of data and materials}

The datasets used and/or analyzed during the current study are available from the corresponding author on reasonable request
Ethics approval and consent to participate

This study was approved by the Research Ethics Committee of the Faculty of Medicine at Menoufia University in Egypt on 13 August 2017; reference number of approval: 38/12-17RADIO.

All patients included in this study gave written informed consent to participate in this research.

\section{Consent for publication}

All patients included in this research gave written informed consent to publish the data contained within this study.

\section{Competing interests}

The authors declare that they have no competing interests.

\section{Author details}

'Radiology Department, Faculty of Medicine, Menoufia University, Yassin Abdel-Ghafar Street, Shebin El-Kom, Menoufia, Egypt. ${ }^{2}$ Menouf General Hospital, Menoufia, Egypt.

Received: 2 October 2019 Accepted: 29 December 2019

Published online: 07 January 2020

\section{References}

1. Tchirikov M, Schroder H J, And Hecher K. Ductus venosus shunting in the fetal venous circulation regulatory mechanisms, diagnostic methods and medical importance. Ultrasound Obstet Gynecol. 2006, 27: 452-461.

2. Ebbing C, Rasmussen S, Godfrey KM et al (2009) Redistribution pattern of fetal liver circulation in intrauterine growth restriction. Acta Obstet Gynecol Scand. 88(10):1118-1123

3. Sabria J, Comas C, Barceló-Vidal C et al (2012) Updated reference ranges for the ductus venosus pulsatility index at 11-13 weeks. Fetal Diagn Ther. 32(4):271-276

4. Baschat AA (2011) Examination of the fetal cardiovascular system. Semin Fetal Neonatal Med 16:2-12

5. Crispi F, Gratacós E (2012) Fetal cardiac function: technical considerations and potential research and clinical applications. Fetal Diagn Ther 32:47-64

6. Baschat AA, Turan OM, Turan S (2012) Ductus venosus blood flow patterns - more than meets the eye? Ultrasound Obstet Gynecol 39:598-599

7. Borrell A, Grande M, Bennasar M et al (2013) First-trimester detection of major cardiac defects with the use of ductus venosus blood flow. Ultrasound Obstet Gynecol. 42:51-57

8. Parlakgumus HA, Durukan T (2010) The relationship between cardiac adaptation to uteroplacental Doppler flow and perinatal outcome in pregnant women with diabetes. Clin Exp Obstet Gynecol. 37:39-42

9. Flood K, Malone FD (2008) Screening for fetal abnormalities with ultrasound. Curr Opin Obstet Gynecol 20:139-145

10. Kiserud T (1999) Hemodynamics of the ductus venosus. Eur J Obstet Gynecol Reprod Biol 84:139-147

11. Kiserud T (2000) Fetal venous circulation - an update on hemodynamics. J Perinat Med 28:90-96

12. Baschat AA (2003) Relationship between placental blood flow resistance and precordial venous Doppler indices. Ultrasound Obstet Gynecol 22: 561-566

13. Bellotti M, Pennati G, De Gasperi C et al (2004) Simultaneous measurements of umbilical venous, fetal hepatic, and ductus venosus blood flow in growth-restricted human fetuses. Am J Obste Gynecol 190:1347-1358

14. Frusca T, Todros T, Lee C et al (2018) Outcome in early-onset fetal growth restriction is best combining computerized fetal heart rate analysis with ductus venosus Doppler: insights from the Trial of Umbilical and Fetal Flow in Europe. Am J Obstet Gynecol 218(2):S783-S789

15. Bahlmann F, Wellek S, Reinhardt I et al (2000) Reference values of ductus venosus flow velocities and calculated waveform indices. Prenat Diagn. 20(8):623-634

16. Axt-Fliedner R, Diler S, Georg T et al (2004) Reference values of ductus venosus blood flow velocities and waveform indices from 10 to 20 weeks of gestation. Arch Gynecol Obstet 269:199-204

17. Kessler J, Rasmussen S, Hanson M, Kiserud T (2006) Longitudinal reference ranges for ductus venosus flow velocities and waveform indices. Ultrasound Obstet Gynecol 28:890-898 
18. Tongprasert F, Srisupundit K, Luewan S, Wanapirak C et al (2012) Normal reference ranges of ductus venosus Doppler indices in the period from 14 to 40 weeks' gestation. Gynecol Obstet Invest 73:32-37

19. Pokharel P, Alam AM (2017) Fetal ductus venosus pulsatility index and diameter during second and third trimester of gestation. J Nepal Med Assoc 56(205):124-131

\section{Publisher's Note}

Springer Nature remains neutral with regard to jurisdictional claims in published maps and institutional affiliations.

Submit your manuscript to a SpringerOpen ${ }^{\circ}$ journal and benefit from:

- Convenient online submission

- Rigorous peer review

- Open access: articles freely available online

High visibility within the field

- Retaining the copyright to your article

Submit your next manuscript at $\boldsymbol{\nabla}$ springeropen.com 